\title{
Analyse paradigmatique d'un corpus de proverbes à l'aide des matrices de concepts
}

\author{
Paul Franceschi \\ Université de Corse \\ www.univ-corse.fr/ franceschi
}

Article paru dans Semiotica (2007), volume 167, pages 271-282.

\begin{abstract}
In Franceschi (2002), I described a theory based on matrices of concepts that aims at constituting an alternative to the classification proposed by Greimas, in the field of paradigmatic analysis. I set out here to apply the matrices of concepts to the study of a corpus of Corsican proverbs. I recall briefly, first, the framework of matrices of concepts. I further describe the structure of proverbial theses, and I expose then the results of the corresponding analysis.
\end{abstract}

Résumé. Dans Franceschi (2002), j'ai présenté une théorie basée sur les matrices de concepts qui se propose de constituer une alternative à la classification proposée par Greimas, dans le domaine de l'analyse paradigmatique. Je m'attache ici à appliquer les matrices de concepts à l'analyse d'un corpus constitué de proverbes corses. Je rappelle tout d'abord brièvement le modèle des matrices de concepts. Je décris également la structure des thèses proverbiales, avant de présenter les résultats de l'analyse correspondante.

Dans ce qui suit, je m'attacherai à effectuer une analyse paradigmatique d'un corpus de proverbes corses à l'aide des matrices de concepts décrites dans Franceschi (2002).

\section{Les matrices de concepts}

De manière préliminaire, il est utile de décrire l'essentiel du cadre formel qui a été décrit de manière détaillée dans Une classe de concepts (2002). Soit tout d'abord une dualité donnée. Celle-ci peut être dénotée par A/Ā. $\hat{A}$ ce stade, $\mathrm{A}$ et $\overline{\mathrm{A}}$ constituent des concepts duaux. On peut considérer également que $\mathrm{A}$ et $\overline{\mathrm{A}}$ sont des concepts qui possèdent une composante contraire $c$, de sorte que $c[\mathrm{~A}]=1$ et $c[\overline{\mathrm{A}}]=-1$. De même, la polarité $p$ de $\mathrm{A}$ et $\overline{\mathrm{A}}$ étant neutre $(p=0)$, ces derniers concepts peuvent être dénotés par $\mathrm{A}^{0}$ et $\overline{\mathrm{A}}^{0}$.

À ce stade, on peut définir la classe des pôles canoniques. Il suffit de considérer l'extension de la classe précédente $\left\{\mathrm{A}^{0}, \overline{\mathrm{A}}^{0}\right\}$, telle que $\mathrm{A}^{0}$ et $\overline{\mathrm{A}}^{0}$ admettent respectivement un concept corrélatif dont la polarité est soit positive $(p=1)$ soit négative $(p=-1)$. Les concepts qui en résultent peuvent être dénotés respectivement $\operatorname{par}\left\{\mathrm{A}^{+}, \mathrm{A}^{-}\right\}$et $\left\{\overline{\mathrm{A}}^{+}, \overline{\mathrm{A}}^{-}\right\}$. Ainsi, pour une dualité donnée $\mathrm{A} / \overline{\mathrm{A}}$, on obtient les six concepts suivants : $\left\{\mathrm{A}^{+}, \mathrm{A}^{0}\right.$, $\left.\mathrm{A}^{-}, \overline{\mathrm{A}}^{+}, \overline{\mathrm{A}}^{0}, \overline{\mathrm{A}}^{-}\right\}$. Appelons-les les pôles canoniques. Ceci conduit à distinguer entre les pôles canoniques positifs $\left(\mathrm{A}^{+}, \overline{\mathrm{A}}^{+}\right)$, neutres $\left(\mathrm{A}^{0}, \overline{\mathrm{A}}^{0}\right)$ et négatifs $\left(\mathrm{A}^{-}, \overline{\mathrm{A}}^{-}\right)$. Enfin, la classe constituée par les six pôles canoniques peut être dénommée la matrice canonique : $\left\{\mathrm{A}^{+}, \mathrm{A}^{0}, \mathrm{~A}^{-}, \overline{\mathrm{A}}^{+}, \overline{\mathrm{A}}^{0}, \overline{\mathrm{A}}^{-}\right\}$.

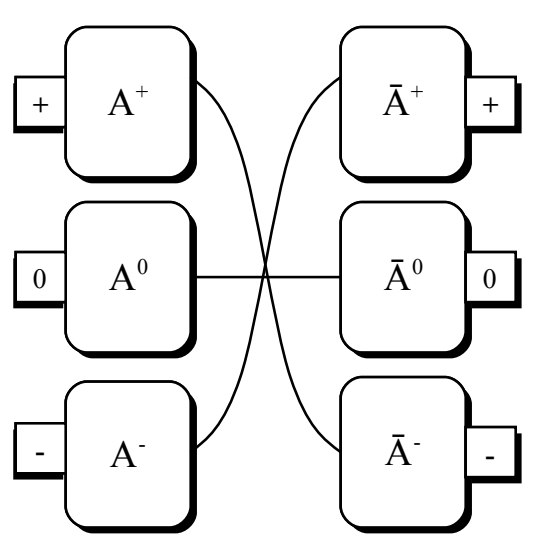

Figure 1 
Intéressons-nous maintenant aux relations existant entre les pôles canoniques d'une même matrice. Parmi les relations existant entre les six pôles canoniques $\left(\mathrm{A}^{+}, \mathrm{A}^{0}, \mathrm{~A}^{-}, \overline{\mathrm{A}}^{+}, \overline{\mathrm{A}}^{0}, \overline{\mathrm{A}}^{-}\right)$, on peut mentionner : dualité, antinomie, complémentarité, corollarité, connexité, anti-connexité. Ainsi, deux pôles canoniques donnés d'une même matrice sont :

(i) duaux si leurs composantes contraires sont opposées et leurs polarités sont neutres

(ii) contraires (ou antinomiques) si leurs composantes contraires sont opposées et leurs polarités sont non neutres et opposées

(iii) complémentaires si leurs composantes contraires sont opposées et leurs polarités sont non neutres et égales

(iv) corollaires si leurs composantes contraires sont égales et leurs polarités sont non neutres et opposées

(v) connexes si leurs composantes contraires sont égales et la valeur absolue de la différence de leurs polarités est égale à 1

(vi) anti-connexes si leurs composantes contraires sont opposées et la valeur absolue de la différence de leurs polarités est égale à 1

Résumons : $\left\{\mathrm{A}^{0}, \overline{\mathrm{A}}^{0}\right\}$ sont duaux, $\left\{\mathrm{A}^{+}, \overline{\mathrm{A}}^{-}\right\}$et $\left\{\mathrm{A}^{-}, \overline{\mathrm{A}}^{+}\right\}$sont contraires, $\left\{\mathrm{A}^{+}, \overline{\mathrm{A}}^{+}\right\}$et $\left\{\mathrm{A}^{-}, \overline{\mathrm{A}}^{-}\right\}$sont complémentaires, $\left\{\mathrm{A}^{+}, \mathrm{A}^{-}\right\}$et $\left\{\overline{\mathrm{A}}^{+}, \overline{\mathrm{A}}^{-}\right\}$sont corollaires, $\left\{\mathrm{A}^{0}, \mathrm{~A}^{+}\right\},\left\{\mathrm{A}^{0}, \mathrm{~A}^{-}\right\},\left\{\overline{\mathrm{A}}^{0}, \overline{\mathrm{A}}^{+}\right\}$et $\left\{\overline{\mathrm{A}}^{0}, \overline{\mathrm{A}}^{-}\right\}$sont connexes, $\left\{\mathrm{A}^{0}, \overline{\mathrm{A}}^{+}\right\},\left\{\mathrm{A}^{0}, \overline{\mathrm{A}}^{-}\right\},\left\{\overline{\mathrm{A}}^{0}, \mathrm{~A}^{+}\right\}$et $\left\{\overline{\mathrm{A}}^{0}, \mathrm{~A}^{-}\right\}$sont anti-connexes.

À partir du cadre théorique ainsi défini, on est à même d'obtenir une classification des concepts comportementaux, qui est calquée sur la structure des matrices de concepts. Parmi les concepts que cette dernière classification permet d'appréhender, on peut citer ainsi : courage, paresse, persévérance, entêtement, prodigalité, dogmatisme, modestie, etc. À titre d'exemple, la matrice à laquelle correspond le concept de courage est la suivante (une énumération plus générale de la classe des concepts comportementaux est présentée dans Franceschi (2002, note 22) :

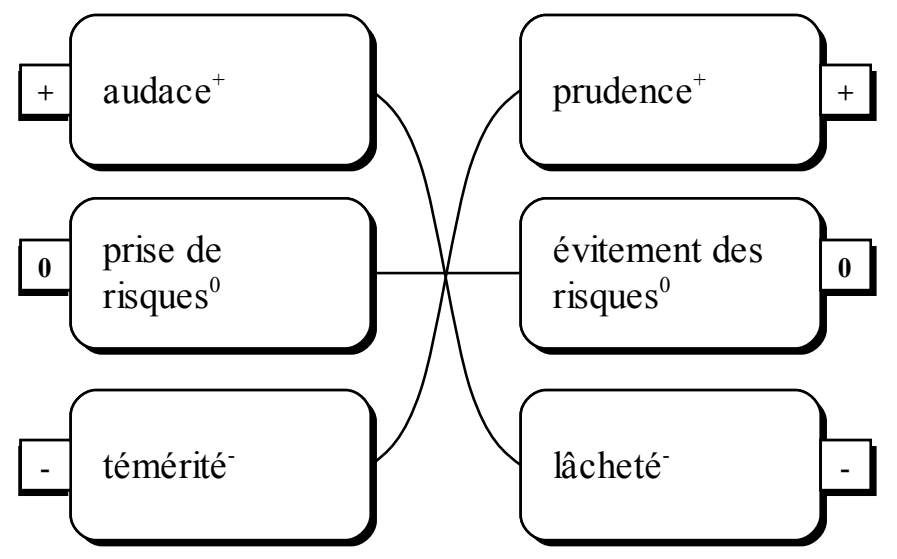

Figure 2

Cette classification des concepts comportementaux servira de base à la présente étude. Mais avant de présenter les résultats auxquels conduit cette dernière, il est nécessaire de décrire plus en détail la structure particulière des propositions proverbiales qui font l'objet de la présente analyse.

\section{Structure des thèses proverbiales}

Dans ce qui suit, chacun des proverbes étudiés sera considéré comme une thèse simple (Franceschi 2004) relative à un concept d'une matrice donnée. D'une manière générale, une thèse simple constitue une appréciation - négative, neutre ou positive - formulée pare rapport à un concept donné. Si l'on dénote par $\alpha$ un tel concept, la structure de la thèse simple correspond alors à $\zeta^{\mathrm{p}}(\alpha)$, avec $\alpha \in\left\{\mathrm{A}^{+}, \mathrm{A}^{0}, \mathrm{~A}^{-}, \overline{\mathrm{A}}^{+}, \overline{\mathrm{A}}^{0}, \overline{\mathrm{A}}^{-}\right\}, p$ dénotant une polarité positive $(p=1)$, neutre $(p=0)$ ou négative $(p=-1)$. Une appréciation négative s'assimile alors à un blâme et une appréciation positive à un éloge (l'appréciation neutre, qui se rencontre rarement, peut être ignorée ici). Ainsi, le blâme d'un concept $\alpha$ est dénoté par $\zeta^{\zeta}(\alpha)$ et son éloge par $\zeta^{+}(\alpha)$. Les différents cas de figure théoriques, par rapport aux six concepts d'une même matrice, sont ainsi les suivants : $\zeta^{-}\left(\mathrm{A}^{+}\right), \zeta\left(\mathrm{A}^{0}\right), \zeta\left(\mathrm{A}^{-}\right), \zeta\left(\overline{\mathrm{A}}^{+}\right), \zeta\left(\overline{\mathrm{A}}^{0}\right), \zeta\left(\overline{\mathrm{A}}^{-}\right), \zeta^{+}\left(\mathrm{A}^{+}\right), \zeta^{+}\left(\mathrm{A}^{0}\right), \zeta^{+}\left(\mathrm{A}^{-}\right), \zeta^{+}\left(\overline{\mathrm{A}}^{+}\right), \zeta^{+}\left(\overline{\mathrm{A}}^{0}\right), \zeta^{+}\left(\overline{\mathrm{A}}^{-}\right)$. 
Il convient maintenant de s'intéresser aux thèses proverbiales simples, qui présentent la structure d'un blâme ou d'un éloge. Considérons tout d'abord le blâme. Un certain nombre de thèses proverbiales comportent ainsi une appréciation dévalorisante, dépréciative, par rapport à un certain type de comportement, une manière d'agir ou d'appréhender les choses. On peut dénoter ce type de thèse simple par $\zeta(s)$ où $s$ désigne un type de comportement ou une manière de considérer les choses. Pour fixer les idées, considérons quelques exemples. Soit la thèse proverbiale suivante :

(1) À chì dormi un piglia pesci. (Celui qui dort ne prend pas de poissons)

Le point de vue exprimé ici est que l'inactivité ou la paresse ne permettent pas à une personne de subvenir à ses propres besoins, de se nourrir. Ceci s'analyse en un jugement négatif, dépréciatif vis-à-vis de la paresse. Ce dernier concept peut être considéré comme une notion péjorative, que l'on peut dénoter par paresseAinsi, la thèse proverbiale simple exprimée ici présente-t-elle la structure d'un blâme de la paresse-, qui peut être dénotée par $\zeta^{-}$(paresse-).

Soit également cette autre thèse proverbiale :

(2) Un si pò fà u passu più longu chè l'infurcatura. (On ne peut pas faire le pas plus long que l'enfourchure)

Le contenu de cette dernière thèse proverbiale s'analyse comme une appréciation péjorative formulée à l'encontre de la tendance à vouloir faire des choses au-delà de ses propres capacités, à voir trop grand. Une telle thèse présente ainsi une structure qui constitue le blâme du concept péjoratif de vanité, de prétention, que l'on peut ainsi dénoter par $\zeta^{-}$(prétention').

Considérons, en second lieu, les thèses proverbiales qui présentent la structure d'un éloge. De telles thèses comportent une appréciation flatteuse par rapport à un comportement, une propension à agir, une situation ou une manière d'appréhender les choses. On dénote les propositions correspondantes par $\zeta^{+}(s)$ où $s$ désigne une façon de considérer les choses ou un comportement donnés. Considérons ainsi quelques exemples. Soit tout d'abord la thèse proverbiale suivante :

(3) U megliu oru hè quiddu chì piega. (Le meilleur or est celui qui plie)

La structure sémantique de cette thèse proverbiale simple est basée sur une analogie. L'or le plus souple, le plus ductile, est qualifié de «meilleur». Par analogie, cette dernière qualité est étendue aux humains, valorisant ainsi celui qui est capable de faire preuve de souplesse, d'adaptabilité. Il s'agit ainsi d'un éloge, d'une louange formulée vis-à-vis de la souplesse. Cette dernière notion étant méliorative, le point de vue ainsi exprimé présente la structure d'un éloge de la souplesse ${ }^{+}$, soit formellement $\zeta^{+}$(souplesse ${ }^{+}$).

On rencontre également un type de structure identique, au niveau de la thèse suivante :

(4) Chì va pianu va sanu è chì va sanu va luntanu. (Celui qui va lentement va sûrement et celui qui va sûrement va loin)

qui s'analyse comme la valorisation d'une façon d'agir tranquille et non précipitée, c'est-à-dire un éloge du calme $^{+}$, soit de manière formelle $\zeta^{+}\left(\right.$calme $\left.^{+}\right)$.

À ce stade, on est à même de déterminer la valeur de vérité de chacune des thèses simples. La valeur de vérité de chaque type d'éloge ou de blâme $e^{l}$ indique si l'appréciation et le comportement sur lequel elle porte se révèlent cohérents ou non au niveau de la thèse proverbiale considérée, sachant que l'éloge d'un concept positif est vrai, de même que le blâme d'un concept négatif. À l'inverse, l'éloge d'un concept négatif ou neutre et le blâme d'un concept positif ou neutre sont faux. Ainsi, parmi les différents cas qui viennent d'être énumérés, ceux dont la valeur de vérité est vrai sont : $\zeta^{-}\left(\mathrm{A}^{-}\right), \zeta^{-}\left(\overline{\mathrm{A}}^{-}\right), \zeta^{+}\left(\mathrm{A}^{+}\right), \zeta^{+}\left(\overline{\mathrm{A}}^{+}\right)$. Et ceux dont la valeur de vérité est faux sont : $\zeta^{-}\left(\mathrm{A}^{+}\right), \zeta^{-}\left(\mathrm{A}^{0}\right), \zeta\left(\overline{\mathrm{A}}^{+}\right), \zeta^{-}\left(\overline{\mathrm{A}}^{0}\right), \zeta^{+}\left(\mathrm{A}^{0}\right), \zeta^{+}\left(\mathrm{A}^{-}\right), \zeta^{+}\left(\overline{\mathrm{A}}^{0}\right), \zeta^{+}\left(\overline{\mathrm{A}}^{-}\right)$.

On peut mentionner enfin, que l'on peut rencontrer, plus rarement, une structure qui est celle d'une thèse 2composée (Franceschi 2004), qui comporte des appréciations relatives à deux concepts d'une même matrice et qui peut être définie comme la conjonction de deux thèses simples. La thèse proverbiale suivante constitue l'illustration d'une thèse 2-composée :

\footnotetext{
${ }^{1}$ Comme on l'a vu, l'appréciation neutre se rencontre rarement.
} 
(5) A pratica vinci a grammatica. (La pratique domine la théorie) $\equiv \zeta^{-}\left(\right.$prétention $\left.^{-}\right) \wedge \zeta^{-}\left(\right.$prétention $\left.^{-}\right)$

En effet, cette dernière thèse comporte la mention des concepts duaux d'une même matrice (la pratique et la théorie), et s'analyse ainsi en une thèse qui comporte d'une part l'éloge explicite de la pratique (« La pratique domine la théorie ») et d'autre part le blâme implicite de la théorie ("La théorie est dominée par la pratique »). Soit formellement : $\zeta^{+}\left(\right.$pratique $\left.^{0}\right)$ et $\zeta\left(\right.$ théorie $\left.^{0}\right)$.

\section{Analyse du corpus de proverbes}

Dans ce qui suit, je m'attacherai à appliquer la méthodologie qui vient d'être décrite à un corpus constitué de proverbes corses (Wikiquote 2006). La démarche consistera ainsi à sélectionner les proverbes qui présentent la structure d'une thèse simple (plus rarement, il s'agira d'une thèse 2-composée) telle qu'elle vient d'être définie, à en déterminer le contenu sémantique, et à synthétiser les résultats ainsi obtenus dans un tableau final.

De manière préalable, il est utile de distinguer ici les thèses proverbiales simples portant sur un concept comportemental, des proverbes dont la portée se révèle plus limitée. Un certain nombre de proverbes présentent en effet une connotation locale ou temporelle. Plusieurs proverbes comportent ainsi un prédicat local, qui se réfère par exemple à une localité, un village ou une ville donnés. On peut citer ainsi : Sè Bastelica t'avia u portu, Aiacciu saria l'ortu (Si Bastelica avait un port, Ajaccio n'en serait que le jardin). Une autre catégorie de proverbes se caractérise par la présence d'un prédicat temporel, qui se réfère à un mois ou un jour ou l'autre de l'année. Les proverbes qui appartiennent à cette catégorie présentent un champ d'application qui est limité dans le temps. Parmi ces derniers, on peut citer par exemple : Aqua d'aostu, oliu è mostu (De l'eau en août donne de l'huile et du moût).

À ce stade, il est utile de noter que de nombreux proverbes corses présentent la structure conditionnelle suivante : si <comportement> alors < <ituation>, où <situation $>$ consiste dans la description d'une situation, d'un état de fait, faisant suite à un type de comportement donné. La forme paradigmatique du proverbe corse présentant cette structure particulière est ainsi : À chì (Celui qui) ... <comportement $>\ldots<$ situation $>$.

Une telle structure se révèle particulièrement adaptée à la formulation des thèses simples, compte tenu du fait que <comportement $>$ et <situation $>$ peuvent présenter une forme négative, neutre ou positive. En particulier, la polarité - positive ou négative - attribuée à <situation> permet l'expression de l'éloge ou du blâme relatif à un type de <comportement $>$ donné. Les proverbes ci-dessous constituent l'illustration d'une telle structure :

(1) À chì dormi un piglia pesci. (Celui qui dort ne prend pas de poissons)

(6) À chì posa mal' pensa (Celui qui reste sans rien faire, pense à mal)

On peut mentionner également qu'une telle structure constitue une instance de la forme causale plus générale : si $<$ cause $>$ alors $<$ effet $>$.

À ce stade, on est en mesure de présenter les résultats de l'analyse effectuée à l'aide de la méthodologie basée sur les matrices de concepts qui vient d'être décrite. Tout d'abord, la partie du corpus qui a fait l'objet de l'analyse est constitué par les thèses proverbiales suivantes ${ }^{2}$ :

\footnotetext{
${ }^{2}$ On peut noter ici qu'un certain nombre de matrices de concepts comportementaux - qui peuvent être énumérées sous forme matricielle simplifiée - n'ont pas été utilisées ici :

$\left\{\right.$ objectivité $^{+}$, être neutre ${ }^{0}$, impersonnalité $^{-}$engagement ${ }^{+}$, être partisan $^{0}$, parti-pris $\left.^{-}\right\}$

$\left\{\right.$ fermeté $^{+}$, propension à réprimer $^{0}$, sévérité, clémence $^{+}$, propension à pardonner $^{0}$, laxisme $^{-}$\}

\{expansion $^{+}$, recherche de la quantité $^{0}$, excès ${ }^{-}$, perfectionnisme ${ }^{+}$, recherche de la qualité ${ }^{0}$, hyper-sélectivité-

\{délicatesse ${ }^{+}$, sensibilité $^{0}$, sensiblerie, , sang-froid ${ }^{+}$, impassibilité $^{0}$, froideur $^{-}$\}

droiture $^{+}$, agir de façon directe ${ }^{0}$, brusquerie ${ }^{-}$, tact $^{+}$, agir de façon indirecte ${ }^{0}$, propension à biaiser ${ }^{-}$\}

\{combativité , goût de l'attaque $^{0}$, agressivité, protection $^{+}$, goût de la défense ${ }^{0}$, repli $^{-}$\}

\{mobilité ${ }^{+}$, penchant au déplacement ${ }^{0}$, instabilité, stabilité ${ }^{+}$, attrait du même endroit ${ }^{0}$, sédentarité $^{-}$,

\{sens de l'humour ${ }^{+}$, goût du jeu ${ }^{0}$, légèretée, sérieux ${ }^{+}$, goût de l'activité sérieuse ${ }^{0}$, se prendre au sérieux ${ }^{-}$\}

$\left\{\right.$ discrétion $^{+}$, garder pour soi ${ }^{0}$, inhibition', ouverture $^{+}$, rendre public ${ }^{0}$, indiscrétion $\left.{ }^{-}\right\}$

\{réceptivité ${ }^{+}$, propension à croire ${ }^{0}$, crédulité $^{-}$, scepticisme constructif ${ }^{+}$, propension à douter $^{0}$, scepticisme excessif $^{-}$,

\{optimisme $^{+}$, appréhender les avantages ${ }^{0}$, optimisme béat ${ }^{-}$, méfiance $^{+}$, voir les inconvénients $^{0}$, pessimisme $^{-}$\}

\{sens du collectif ${ }^{+}$, faire comme les autres ${ }^{0}$, conformisme $^{-}$, originalité $^{+}$, se distinguer ${ }^{0}$, excentricité $^{-}$\}

\{résolution ${ }^{+}$, garder une opinion ${ }^{0}$, entêtement', souplesse d'esprit $^{+}$, changer d'avis ${ }^{0}$, versatilité $\}$

\{goût de la liberté ${ }^{+}$, être affranchi ${ }^{0}$, indiscipline-, obéissance ${ }^{+}$, se soumettre à une règle ${ }^{0}$, servilité $\}$

\{réflexion ${ }^{+}$, intériorisation ${ }^{0}$, inhibition ${ }^{-}$, sociabilité ${ }^{+}$, extériorisation $^{0}$, sans-gêne $^{-}$\}
} 
(7) A lingua ossu ùn ha è ossu tronca. (La langue ne possède pas d'os, mais peut faire rompre les os) $\equiv \zeta$ (dénigrement')

(8) A misura ancu ind'è l'aqua. (Il faut de la mesure en tout, même lorsqu'on boit) $\equiv \zeta^{+}\left(\right.$modération $\left.^{+}\right)$

(5) A pratica vinci a grammatica. (La pratique domine la théorie) $\equiv \zeta^{+}$(goût pour la pratique $\left.{ }^{0}\right) \wedge \zeta^{-}$(goût pour la théorie ${ }^{0}$ )

(9) A regula ci stà bè ancu in casa di u rè. (Même dans la maison du roi, on fait des économies) $\equiv \zeta^{+}$ (sens de l'économie ${ }^{-}$)

(10) A a fica zemba ognunu s'arremba. (Tout le monde profite du faible. Litt. : tout le monde s'appuie sur le fichier qui penche) $\equiv \zeta$ (faiblesse')

(11) À chì campa spirendu mori caghendu. (Celui qui vit d'espérance, meurt dans le dénuement) $\equiv \zeta$ (attrait pour les chimères')

(12) $\grave{A}$ chi di piombu tomba di piombu mori. (Celui qui tue par le plomb périt par le plomb) $\equiv \zeta$ (violence-)

(1) À chì dormi un piglia pesci. (Celui qui dort ne prend pas de poissons) $\equiv \zeta^{-}$(paresse')

(13) À chì l'attempa a perdi. (Celui qui diffère une chose, finit par la perdre) $\equiv \zeta$ (lenteur)

(14) À chì muta muga. (Celui qui fait des changements, blesse) $\equiv \zeta$ (inclination au changement $\left.{ }^{0}\right)$

(6) À chì posa mal' pensa (Celui qui reste sans rien faire, pense à mal) $\equiv \zeta$ (paresse-)

(15) À chì s'aiuta hè galant' omu. (Il est louable de se prendre en main) $\equiv \zeta^{+}$(s'occuper de soi)

(16) À chì servi u cumunu ùn servi à nissunu. (Celui qui se sert le bien commun ne sert personne) $\equiv \zeta$ (altruisme $^{+}$)

(17) À chì tropppu abbraccia nudda strigni. (Qui trop embrasse mal étreint) $\equiv \zeta$ (dispersion-)

(18) À chi tropppu si cala, u culu vi mostra. (Celui qui se baisse trop, montre son cul) $\equiv \zeta$ (sousestimation de soi')

(19) À chì ùn arrisica ùn arruzica. (Qui ne risque rien n'a rien. Litt. : celui qui ne risque rien n'a rien à ronger) $\equiv \zeta^{-}$(éviter les risques ${ }^{0}$ )

(20) À chì ùn si misura ùn dura. (Celui qui ne fait pas preuve de mesure ne dure pas) $\equiv \zeta$ (immodération')

(4) Chì va pianu va sanu è chì va sanu va luntanu. (Celui qui va lentement va sûrement et celui qui va sûrement va loin $) \equiv \zeta^{+}\left(\right.$calme $\left.^{+}\right)$

(21) Ci voli à lagà u fusu à chì n'ha l'usu. (Chacun son métier. Litt. : Il faut laisser le fuseau à celui dont c'est le métier $) \equiv \zeta\left(\right.$ expertise $\left.{ }^{+}\right)$

(22) Di fà ciò chì hè fattu ùn hè piccatu. (Rendre ce que l'on nous a fait n'est pas pêcher) $\equiv \zeta^{+}$(violence')

(23) I funi longhi diventani sarpi. (Les cordes trop longues deviennent des serpents) $\equiv \zeta$ (lenteur)

(24) I solda ùn venini micca cantendu. (L'argent ne vient pas en chantant) $\equiv \zeta^{-}$(paresse-)

(25) Tuttu lagatu hè persu. (Toutes les occasions qu'on laisse passer sont perdues) $\equiv \zeta$ (réactions différées $\left.{ }^{0}\right)$

(26) U bè di l'avaru u si magna u furfanti. (Le bien de l'avare, c'est le forban qui en profite) $\zeta^{\text {(avarice-) }}$

(27) $U$ cavaddu chi ùn vò bia hè un gattivu zifulà. (Il ne sert à rien de forcer un cheval qui ne veut pas boire $) \equiv \zeta^{-}$(autoritarisme $)$

(3) U megliu oru hè quiddu chì piega. (Celui qui est souple réussit mieux. Litt. : le meilleur or est celui qui plie $) \equiv \zeta^{+}\left(\right.$souplesse $\left.^{+}\right)$

(28) U troppu bè s'arrivolta. (Si on fait trop de bien aux gens, on finit par en retirer des ennuis. Litt. : faire trop de bien finit par nuire $) \equiv \zeta^{-}$(rendre dépendant $\left.{ }^{-}\right)$

(2) Ùn si pò fà u passu più longu chè l'infurcatura. (On ne peut pas faire le pas plus long que l'enfourchure $) \equiv \zeta^{-}$(prétention')

(29) Ún si pò tena i dui pedi in u scarpu. (On ne peut courir deux lièvres à la fois. Litt. : on ne peut mettre les deux pieds dans une seule chaussure $) \equiv \zeta^{-}\left(\right.$dispersion $\left.^{-}\right)$

(30) Una mani lava l'altra è tremindù lavani $u$ visu. (Une main lave l'autre, et les deux lavent le visage) $\equiv$ $\zeta^{+}\left(\right.$altruisme $\left.{ }^{+}\right)$

Et le tableau final qui en résulte est le suivant :

\footnotetext{
$\left\{\right.$ renouveau $^{+}$, intérêt au changement ${ }^{0}$, rupture $^{-}$, préservation des acquis ${ }^{+}$, intérêt au maintien ${ }^{0}$, conservatisme $^{-}$\} $\left\{\right.$ ampleur de vues ${ }^{+}$, goût de la synthèse ${ }^{0}$, survol$^{-}$, précision $^{+}$, goût de l'analyse ${ }^{0}$, se perdre dans les détails ${ }^{-}$\} amour $^{+}$, attraction $^{0}$, affection mièvre-, savoir prendre ses distances ${ }^{+}$, répulsion $^{0}$, haine $^{-}$\}

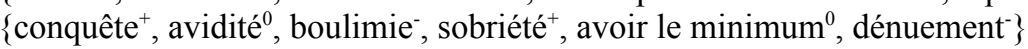




\begin{tabular}{|c|c|c|c|}
\hline & disponibilité $^{+}$ & activité $^{+}$ & \\
\hline & Goût du loisir $^{0}$ & goût du travail $^{0}$ & \\
\hline (1) (6) (24) & paresse & suractivité $^{-}$ & \\
\hline
\end{tabular}

Amour-propre

\begin{tabular}{|c|c|}
\hline & Amour-propre \\
\hline & estime de soi $^{0}$ \\
\hline$(2)$ & prétention $^{-}$ \\
\hline
\end{tabular}

modestie $^{+}$

mise en retrait de l'ego ${ }^{0}$

sous-estimation de soi-

\begin{tabular}{|c|c|c|c|}
\hline$(9)$ & Sens de l'économie $^{+}$ & générosité $^{+}$ & \\
\hline & Inclination à l'épargne $^{0}$ & inclination à la dépense $^{0}$ & \\
\hline$(26)$ & avarice $^{-}$ & prodigalité $^{-}$ & \\
\hline & audace $^{+}$ & prudence $^{+}$ & \\
\hline & Prise de risques $^{0}$ & évitement des risques $^{0}$ & (19) \\
\hline & témérité $^{-}$ & lâcheté $^{-}$ & \\
\hline
\end{tabular}

\begin{tabular}{|c|c|c|c|}
\hline$(15)$ & s'occuper de soi $^{+}$ & altruisme $^{+}$ & $(30)(16)$ \\
\hline & être tourné vers soi-même & & être tourné vers les autres $^{0}$ \\
\hline & égoïsme $^{-}$ & rendre dépendant & $(28)$ \\
\hline
\end{tabular}

\begin{tabular}{|c|c|c|c|}
\hline (3) & souplesse $^{+}$ & constance $^{+}$ & \\
\hline & Tendance à changer $^{0}$ & tendance à ne pas changer $^{0}$ & \\
\hline & inconstance $^{-}$ & rigidité $^{-}$ & \\
\hline
\end{tabular}

\begin{tabular}{|c|c|c|c|}
\hline & idéalisme $^{+}$ & réalisme $^{+}$ & \\
\hline & Appréhender les objectifs $^{0}$ & appréhender les moyens $^{0}$ & \\
\hline$(11)$ & Attrait pour les chimères $^{-}$ & prosaïsme $^{-}$ & \\
\hline
\end{tabular}

\begin{tabular}{|c|c|c|c|}
\hline & Vicacité, spontanéité $^{+}$ & calme $^{+}$ & $(4)$ \\
\hline & Réactions immédiates $^{0}$ & réactions différées $^{0}$ & $(25)$ \\
\hline & précipitation $^{-}$ & lenteur $^{-}$ & $(23)(13)$ \\
\hline
\end{tabular}

\begin{tabular}{|c|c|c|c|}
\hline & Capacité d'abstraction $^{+}$ & pragmatisme $^{+}$ & \\
\hline (5) & goût pour la théorie $^{0}$ & goût pour la pratique $^{0}$ & (5) \\
\hline & dogmatisme & prosaïsme $^{-}$ & \\
\hline
\end{tabular}

\begin{tabular}{|c|c|c|c|}
\hline & sens de l'autorité & sens de la discipline $^{+}$ & \\
\hline & goût du commandement $^{0}$ & obéir $^{0}$ & \\
\hline$(27)$ & autoritarisme & servilité $^{-}$ & \\
\hline
\end{tabular}

\begin{tabular}{|c|c|c|c|}
\hline & défense $^{+}$ & pacifisme $^{+}$ & \\
\hline & refus $^{0}$ & acceptation $^{0}$ & \\
\hline (12) (22) & violence $^{-}$ & faiblesse $^{-}$ & \\
\hline
\end{tabular}

\begin{tabular}{|c|c|c|c|}
\hline & causticité $^{+}$ & valorisation $^{+}$ & \\
\hline & esprit critique $^{0}$ & souligner les qualités $^{0}$ & \\
\hline$(7)$ & dénigrement $^{-}$ & angélisme $^{-}$ & \\
\hline
\end{tabular}

\begin{tabular}{|c|c|c|c|}
\hline & éclectisme $^{+}$ & expertise $^{+}$ & $(21)$ \\
\hline & pluridisciplinarite $^{0}$ & mono-disciplinarité $^{0}$ & \\
\hline (29) (17) & dispersion & cloisonnement $^{-}$ & \\
\hline
\end{tabular}

\begin{tabular}{|c|c|c|c|}
\hline & stabilité $^{+}$ & adaptabilité $^{+}$ & \\
\hline & inclination au statu quo ${ }^{0}$ & inclination au changement $^{0}$ & (14) \\
\hline & invariabilité ${ }^{-}$ & instabilité $^{-}$ & \\
\hline & motivation $^{+}$ & modération $^{+}$ & $(8)$ \\
\hline & passion $^{0}$ & raison $^{0}$ & \\
\hline (20) & immodération- & tiédeur- & \\
\hline
\end{tabular}




\begin{tabular}{|c|c|c|c|}
\hline & fermeté $^{+}$ & diplomatie $^{+}$ & \\
\hline & ne pas céder & faire des concessions $^{0}$ & \\
\hline & intransigeance $^{-}$ & faiblesse $^{-}$ & $(10)$ \\
\hline
\end{tabular}

On le voit, la présente analyse conduit à mettre en évidence plusieurs points intéressants. Elle permet tout d'abord de souligner un certain nombre de congruences sémantiques. Par exemple, les deux thèses proverbiales (1) Á chì dormi un piglia pesci et (24) I solda ùn venini micca cantendu présentent une structure commune qui est celle du blâme de la paressé. De même, l'analyse a permis d'expliciter les relations entre plusieurs thèses proverbiales (par exemple, (2) Ùn si pò fà u passu più longu chè l'infurcatura et (18) $\dot{A}$ chì tropppu si cala, u culu vi mostra) qui sont co-matricielles. En outre, l'analyse permet de souligner d'éventuelles contradictions telles que l'éloge et le blâme d'un même concept. Tel est notamment le cas pour et (22) Di fà ciò chì hè fattu ùn hè piccatu (éloge de la violence-) et (12) A chì di piombu tomba di piombu mori (blâme de la violence-). Enfin, les thèses proverbiales co-matricielles (29) Ún si pò tena i dui pedi in u scarpu et (21) Ci voli à lagà u fusu à chì n'ha l'usu présentent un cas particulier de convergence sémantique : la première thèse proverbiale constitue le blâme du concept négatif de dispersion, alors que la seconde thèse réside dans l'éloge du concept positif opposé d'expertise ${ }^{+}$.

\section{Conclusion}

On le voit, le type d'analyse paradigmatique qui vient d'être décrit, appliqué à un corpus de proverbes corses, permet d'en préciser, selon une approche méthodique, le contenu sémantique. Ce type d'analyse basé sur les matrices de concepts peut aisément être étendu à d'autres corpus parémiologiques, ou bien utilisé pour effectuer des comparaisons entre plusieurs corpus parémiologiques. Plus généralement, on peut observer que ce type d'analyse peut également être appliqué d'autres types de textes au sein duquel la structure des thèses simples se révèle prédominante. ${ }^{3}$

\section{Références}

Franceschi, Paul (2002). Une classe de concepts. Semiotica, 139 (1-4), 211-226.

-(2003). Le plan dialectique : pour une alternative au paradigme. Semiotica, 146 (1-4), 353-367.

Wikiquote (2006). Corsican proverbs, http ://en.wikiquote.org/wiki/Corsican_proverbs

\footnotetext{
${ }^{3}$ Je remercie Françoise Albertini et Dominique Salini pour leurs commentaires concernant le mémoire de DEA Langue et culture corses qui est à l'origine du présent article.
} 
